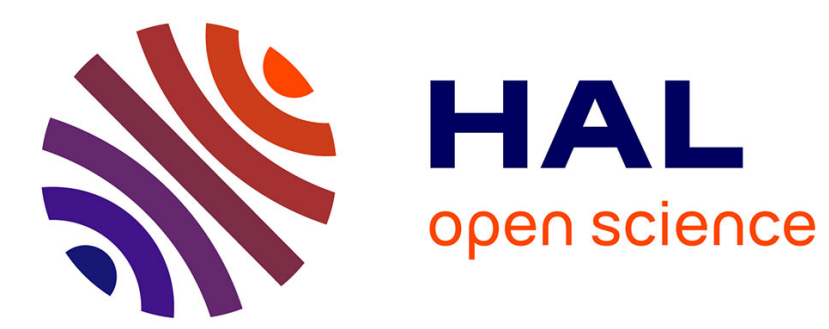

\title{
Sur les traces d'Henri Gaussen en Andorre Jean-Paul Métailié
}

\section{To cite this version:}

Jean-Paul Métailié. Sur les traces d'Henri Gaussen en Andorre. Revue Géographique des Pyrénées et du Sud-Ouest, 1991, 62 (2), pp.139-150. hal-01496423

\section{HAL Id: hal-01496423 \\ https://hal-univ-tlse2.archives-ouvertes.fr/hal-01496423}

Submitted on 27 Mar 2017

HAL is a multi-disciplinary open access archive for the deposit and dissemination of scientific research documents, whether they are published or not. The documents may come from teaching and research institutions in France or abroad, or from public or private research centers.
L'archive ouverte pluridisciplinaire HAL, est destinée au dépôt et à la diffusion de documents scientifiques de niveau recherche, publiés ou non, émanant des établissements d'enseignement et de recherche français ou étrangers, des laboratoires publics ou privés. 


\title{
Sur les traces d'Henri Gaussen en Andorre
}

\author{
par Jean-Paul MÉTAILIE *
}

Henri Gaussen, a laissé à sa mort en 1981, une collection photographique de plusieurs milliers de clichés couvrant le monde entier et une période allant du début du siècle aux années 1970 : elle est en cours d'inventaire et d'analyse. Phytogéographe, botaniste, pionnier de la cartographie de la végétation et fondateur de nombreux organismes scientifiques, Henri Gaussen resta toute sa vie un "piéton des Pyrénées » et il n'avait cessé de parcourir de long en large ces vallées qu'il aimait, l'appareil photographique en bandoulière. La partie ancienne de sa collection, étalée du début du siècle aux années 1945, constitue aujourd'hui une des principales sources d'archives photographiques sur les Pyrénées, permettant des recherches sur l'histoire récente des paysages et des milieux végétaux. Le fonds ancien consiste en 4431 plaques de verre négatives, 1199 négatifs souples et 2170 plaques de verre positives; des albums de tirages existent également; l'ensemble est conservé aux Archives départementales de la Haute-Garonne.

Arrivé dans les Pyrénées en 1901, à l'âge de 10 ans, Henri Gaussen se passionna immédiatement pour la montagne et se mit à sillonner l'Ariège ; il a lui-même souligné à diverses reprises combien sa vocation phytogéographique avait été liée à cette pratique constante de la montagne. C'est le souvenir de ces multiples randonnées, cette mémoire photographique, qui lui ont permis, en captivité, pendant la guerre de 1914-18, de jeter les bases théoriques et méthodologiques du travail qui allait déboucher sur sa thèse, en 1926, et sur ses recherches ultérieures.

* Chargé de recherches au CNRS, CIMA, Institut Danicl Fauchcr, Universitć de Toulouse--Le Mirail, 31058 Toulouse cedex. 


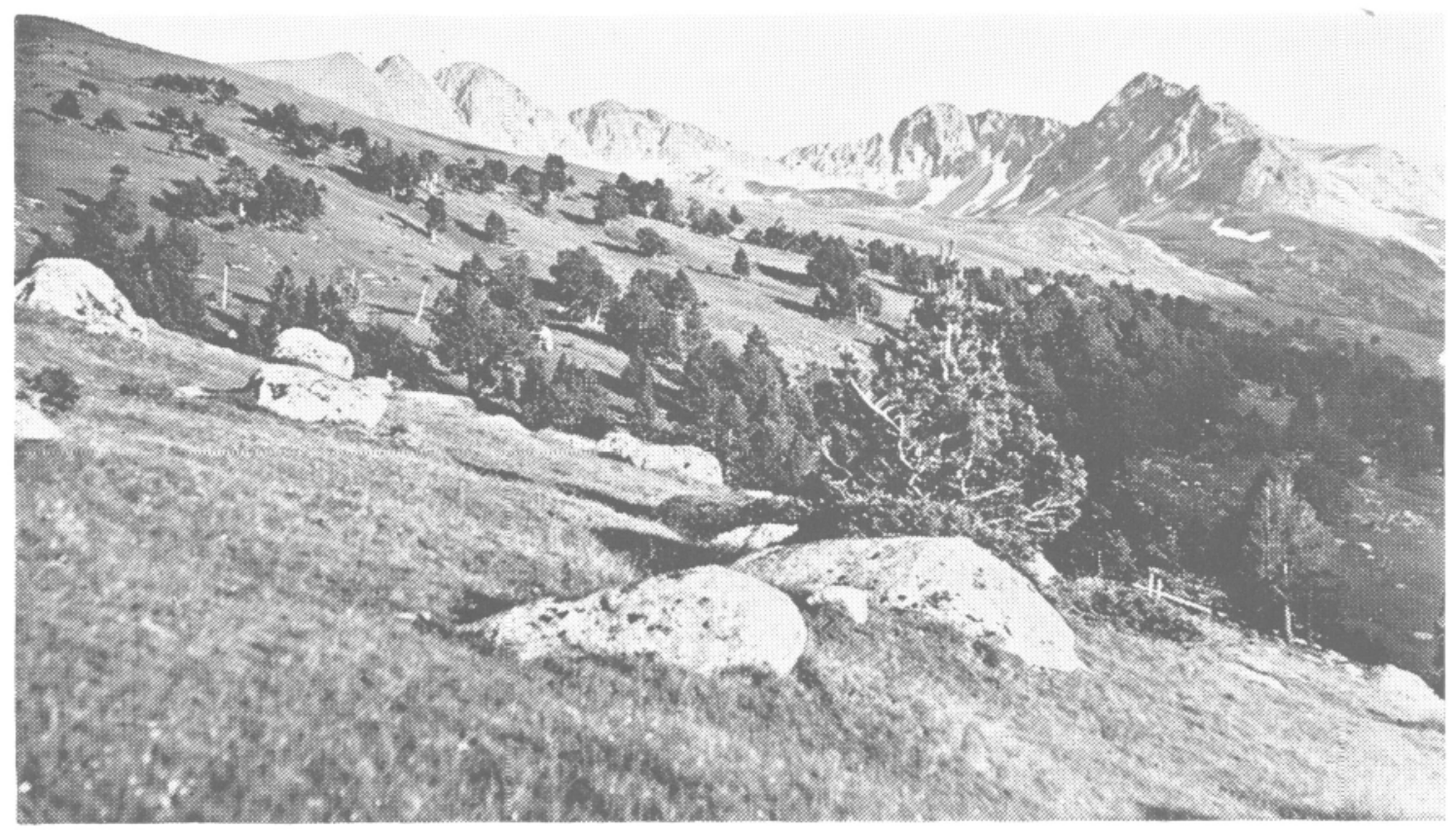

Photographic n` 1: Cirque des Pessons, juillet 1936 (cliché H. Gaussen).

L'Andorre, voisine de l'Ariège, fut naturellement une région que Henri Gaussen fréquenta très tôt, et souvent. Il y fit de nombreuses randonnées avant la guerre, particulièrement dans les années 19101914, puis y revint souvent dans les années 1920-30; les photographies sur l'Andorre de sa collection datent de cette période, les plus anciennes ayant été prises par ses frères aînés. La plupart des clichés ont été tirés le long des deux principaux axes de pénétration : Valira d'Orient et Valira del Nord; on y relève assez peu de vues des milieux d'altitude, mais surtout des paysages de fond de vallée, les villages, les églises. Quelques exemples montrent quel parti l'on peut tirer de ces documents, au fil des itinéraires d'Henri Gaussen, pour tenter de mesurer l'évolution récente des paysages andorrans.

Le premier fait partie d'un panorama du cirque des Pessons pris en juillet 1936 non loin de la route du col d'Envalira, au niveau du premier lacet de cette route. La vue montre la solana de la Costa de les Grandalles, soit un versant exposé au sud, entre 2000 et $2400 \mathrm{~m}$ d'altitude. Il s'agit ici d'un des cortons - unité pastorale utilisée par les troupeaux andorrans ou transhumants - de haute solana, extrêmement pâturé dans le passé par les troupeaux d'ovins transhumants. Encamp et Canillo, dans le haut Valira d'Orient, sont les paroisses qui possèdent les plus vastes cortons de ce type.

Deux faits liés à l'allègement de la pression pastorale sont clairement visibles en comparant ce cliché avec le second, pris en septembre 1991. D'abord, l'accroissement de la biomasse herbacée : la pelouse à Nardus stricta et Festuca rubra, au premier plan, était en 


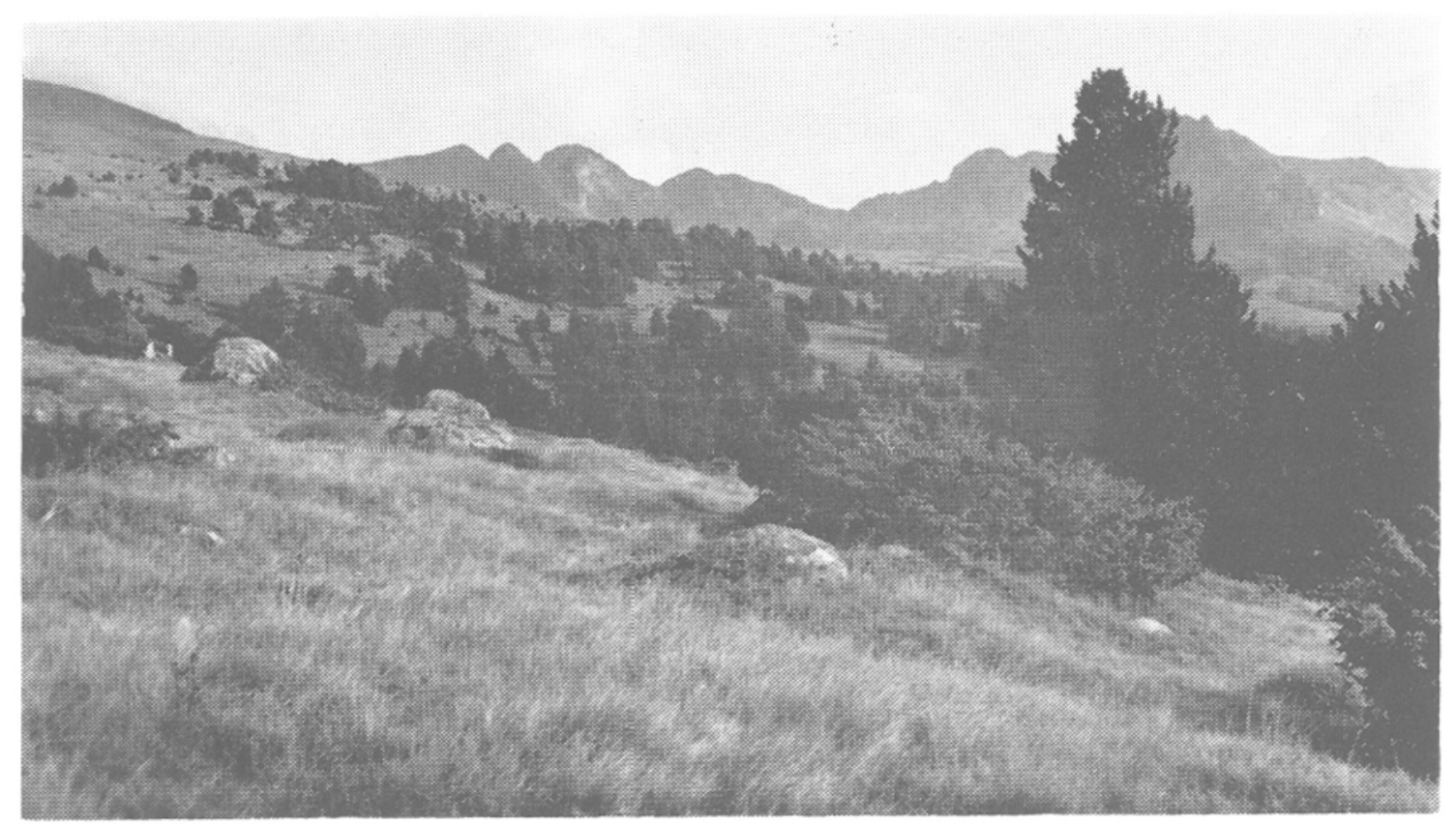

Photographie n 2 : Cirque des Pessons, septembre 1991 (cliché J.P. Métaillé).

1936 tout à fait rase ; aujourd'hui, elle s'épaissit, commence à absorber les blocs erratiques dispersés, selon une dynamique que l'on retrouve maintenant partout dans les pâturages des Pyrénées. Sur les pentes supérieures, la lande pelouse à Festuca eskia et Calluna vulgaris se densifie également, y compris sur les replats. Ensuite, la progression de la pinède : c'est également une dynamique habituelle, que l'on va retrouver dans tous les clichés d'Andorre, mais qui est intéressante ici en raison de la situation marginale des boisements. Les groupes d'arbres assez dispersés qui parsèment le versant en 1936 donnent alors une image de limite forestière résiduelle, en recul, en dépit de l'altitude assez basse pour le pin à crochets $(2100 \mathrm{~m}$ $2200 \mathrm{~m}$ ), qui monte bien plus haut en Andorre. En 1991, un seuil semble avoir été franchi depuis peu: non seulement les bosquets ont grandi et se sont notablement épaissis, mais tout le versant est piqueté d'un semis de jeunes pins de 10 à 20 ans d'âge, qui préfigurent la prochaine recolonisation forestière. On rencontre ces semis sur toute la solana d'Envalira, jusqu'à une altitude de $2400 \mathrm{~m}$; cette colonisation sera vaisemblablement lente en raison des contraintes liées à l'exposition sud: gel, sécheresse, etc., ainsi qu'aux difficultés de régénération que rencontrent les arbres dans les pelouses; mais la pression pastorale ne semble plus constituer le facteur limitant principal sur ce versant depuis une trentaine d'années.

Continuant la descente vers les vallées, on arrive au nivcau des bordes d'été, qui sont ici parmi les plus hautes d'Andorre, puisque l'on se trouve à $2000 \mathrm{~m}$. Le cliché de 1901 fut probablement pris 


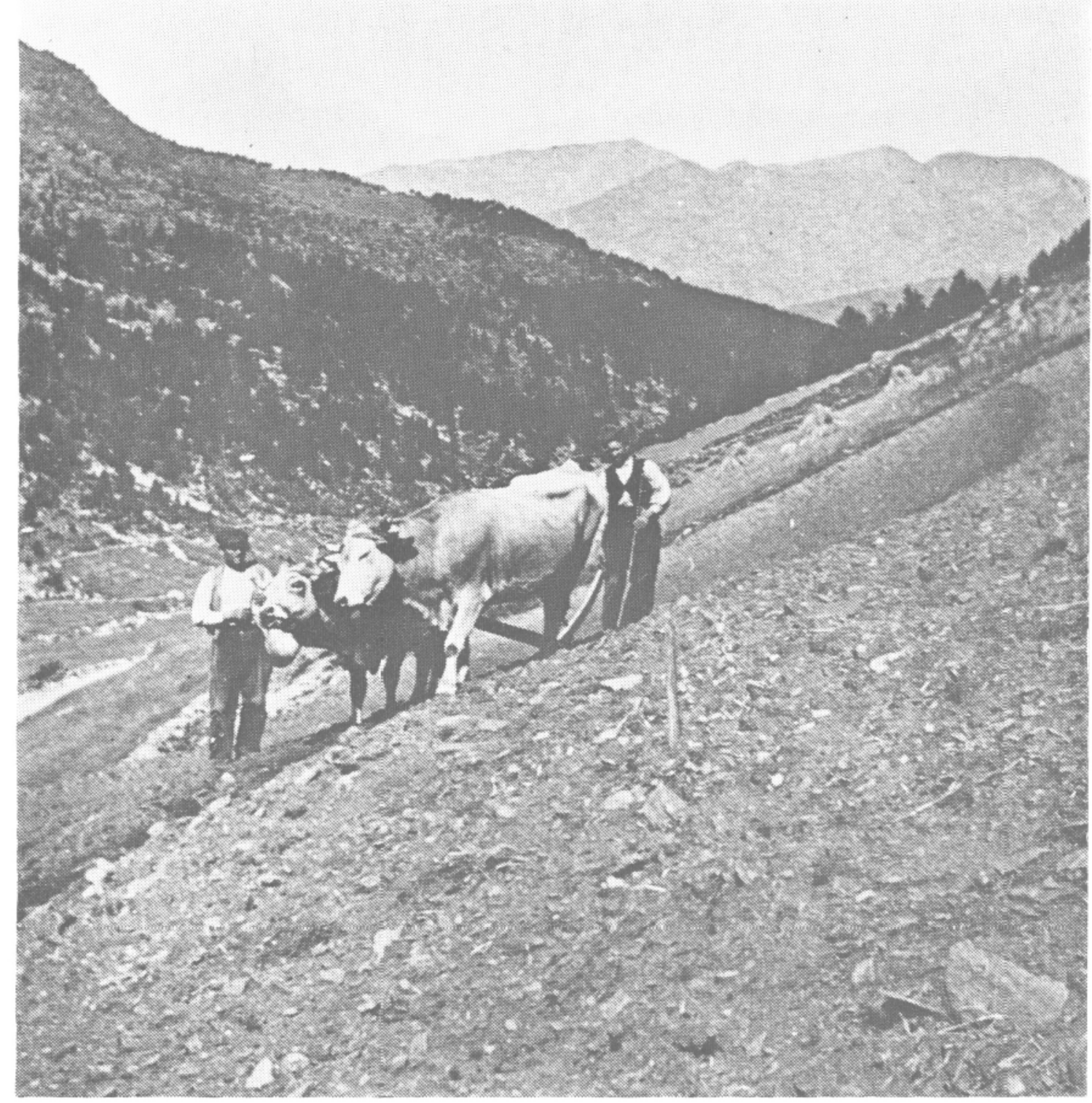

Photographie $\mathrm{n}^{\circ}$ 3: Labours à l'araire, Bordes d'Envalira en amont de Soldeu, 1901 (cliché Charles Gaussen).

en août ou septembre, date qui peut se déduire de la présence des gerbes de moissons visibles au second plan. Les cultures de haute solana, seigle ou pommes de terre, demandaient une jachère d'un an en dépit de la fumure et les labours commençaient souvent avant même que la moisson ne soit finie dans les parcelles voisines. De nos jours, hôtels et appartements ont remplacé les bordes d'été.

Une autre évolution est bien visible à l'arrière-plan : la reconstitution de la forêt de pins de l'obac, versant exposé au nord. Ces forêts servaient autrefois d'estive de fin d'été et restaient en général à la libre disposition des habitants. La densification de la pinède est 


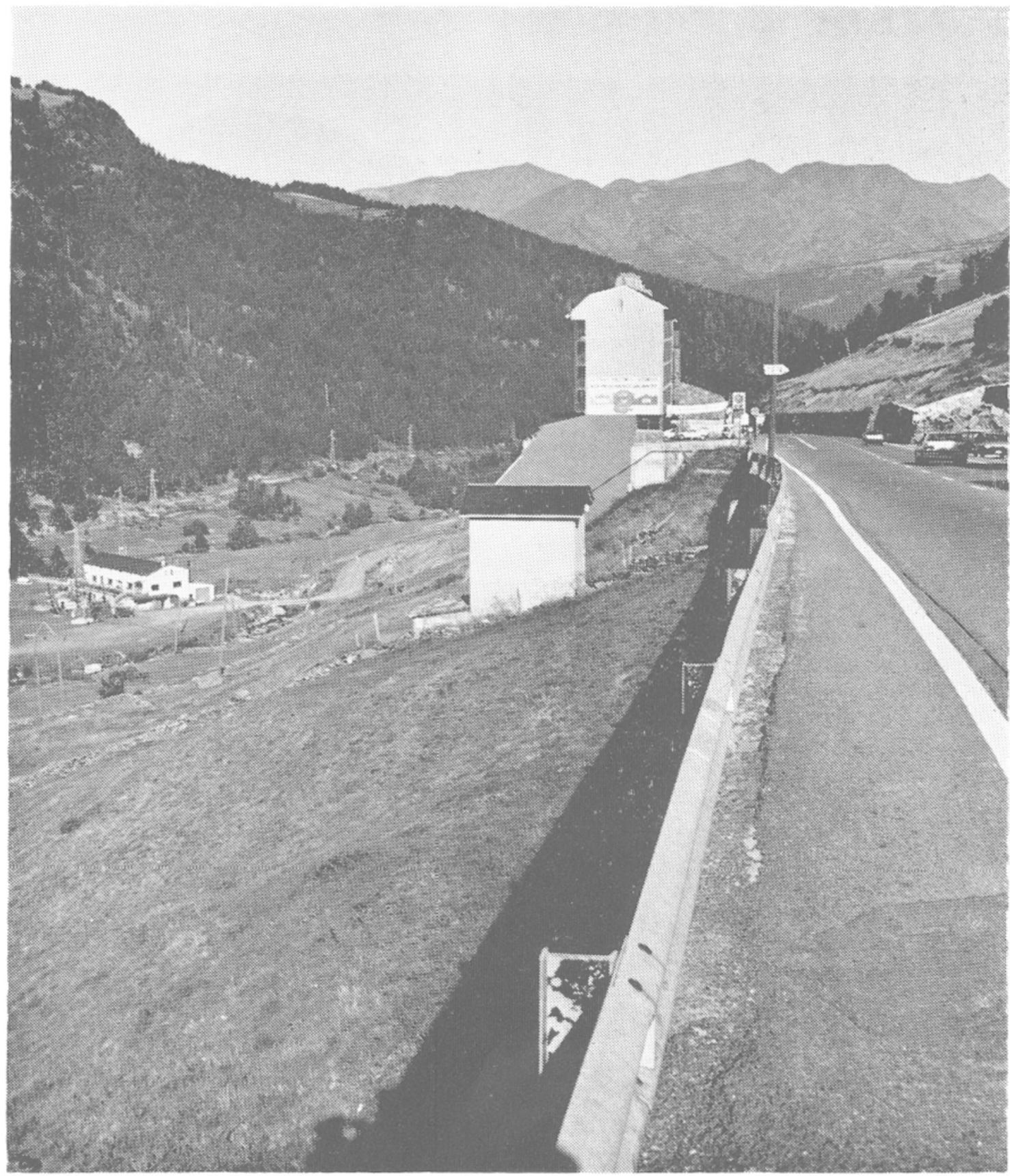

Photographie n’ 4 : Bordes d'Envalira, septembre 1991 (cliché J.P. Métailié).

spectaculaire, comme partout dans les Pyrénées catalanes; la reconquête sur la limite supérieure a fait remonter la forêt de plus de $250 \mathrm{~m}$ en altitude. Il est d'ailleurs possible que la partie supérieure du boisement ait été coupée à blanc quelques années avant la photographie, ce qui est suggéré par sa lisière très nette. Les agressions modernes contre la forêt, invisibles sur la photographie de 1991, 


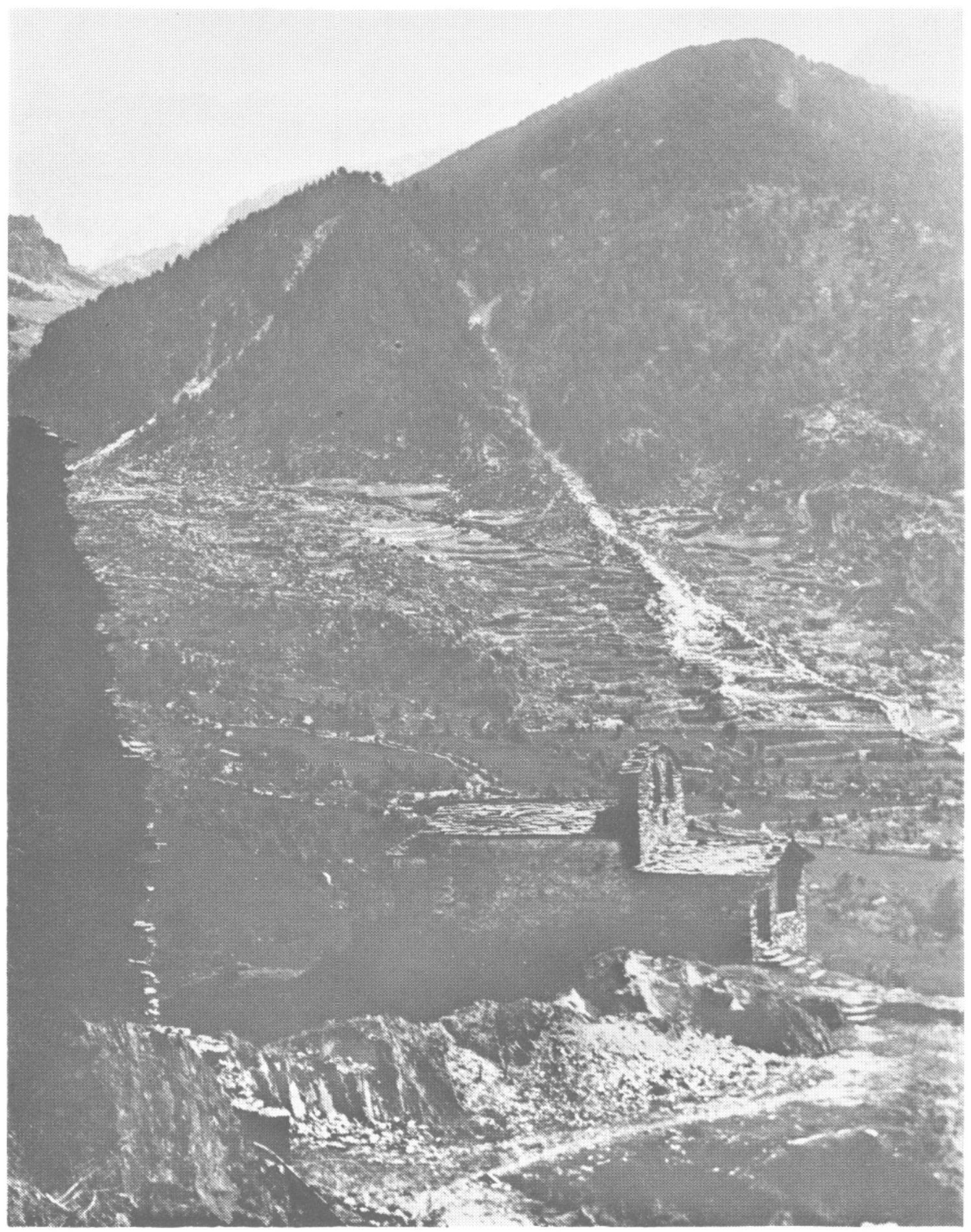

Photographic n" 5: Chapelle de Les Bons, Encamp, septembre 1913 (cliché H. Gaussen). 


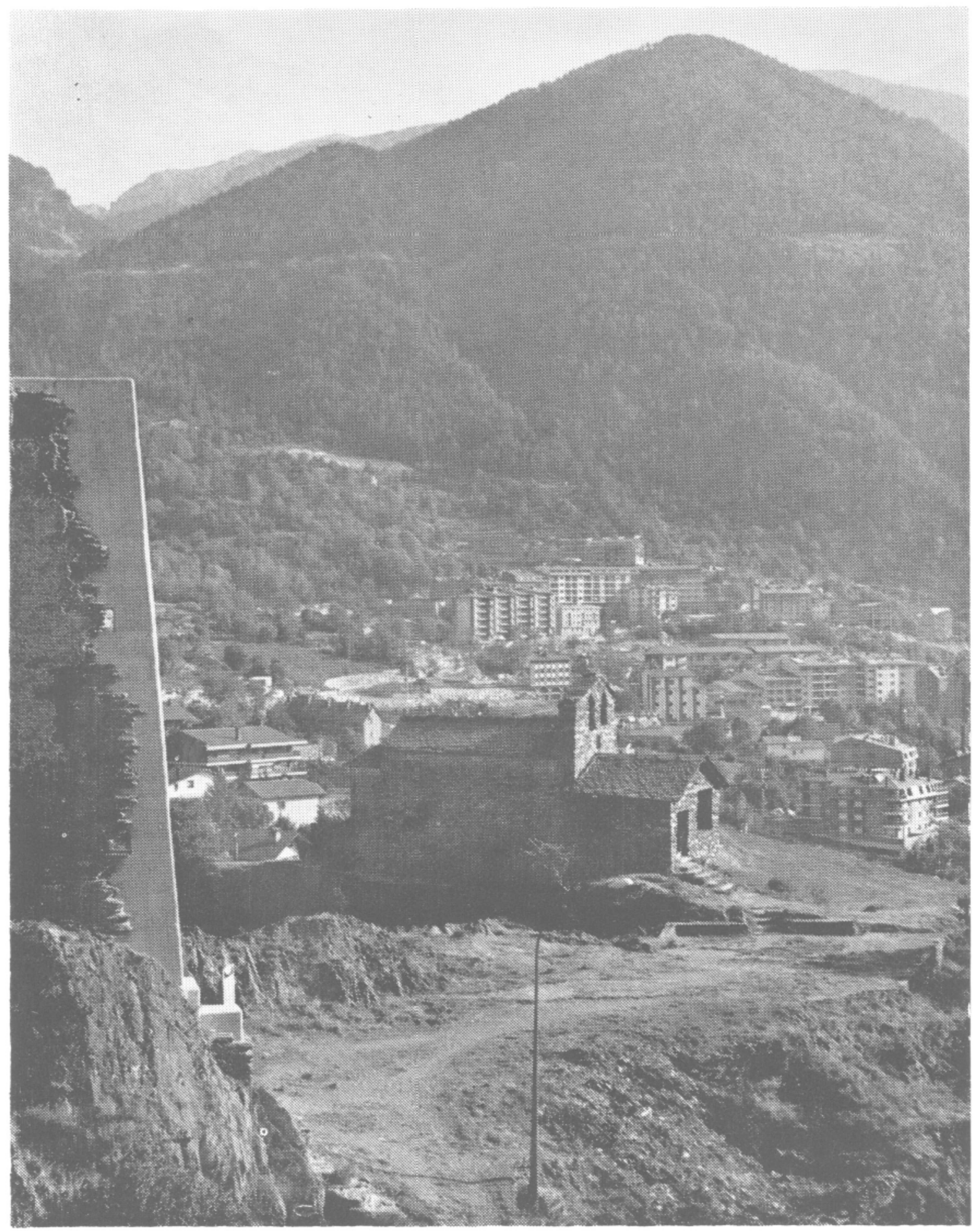

Photographie n ${ }^{\circ}$ 6: Chapelle de Les Bons, Encamp, septembre 1991. (cliché J.-P. Mćtailić). 


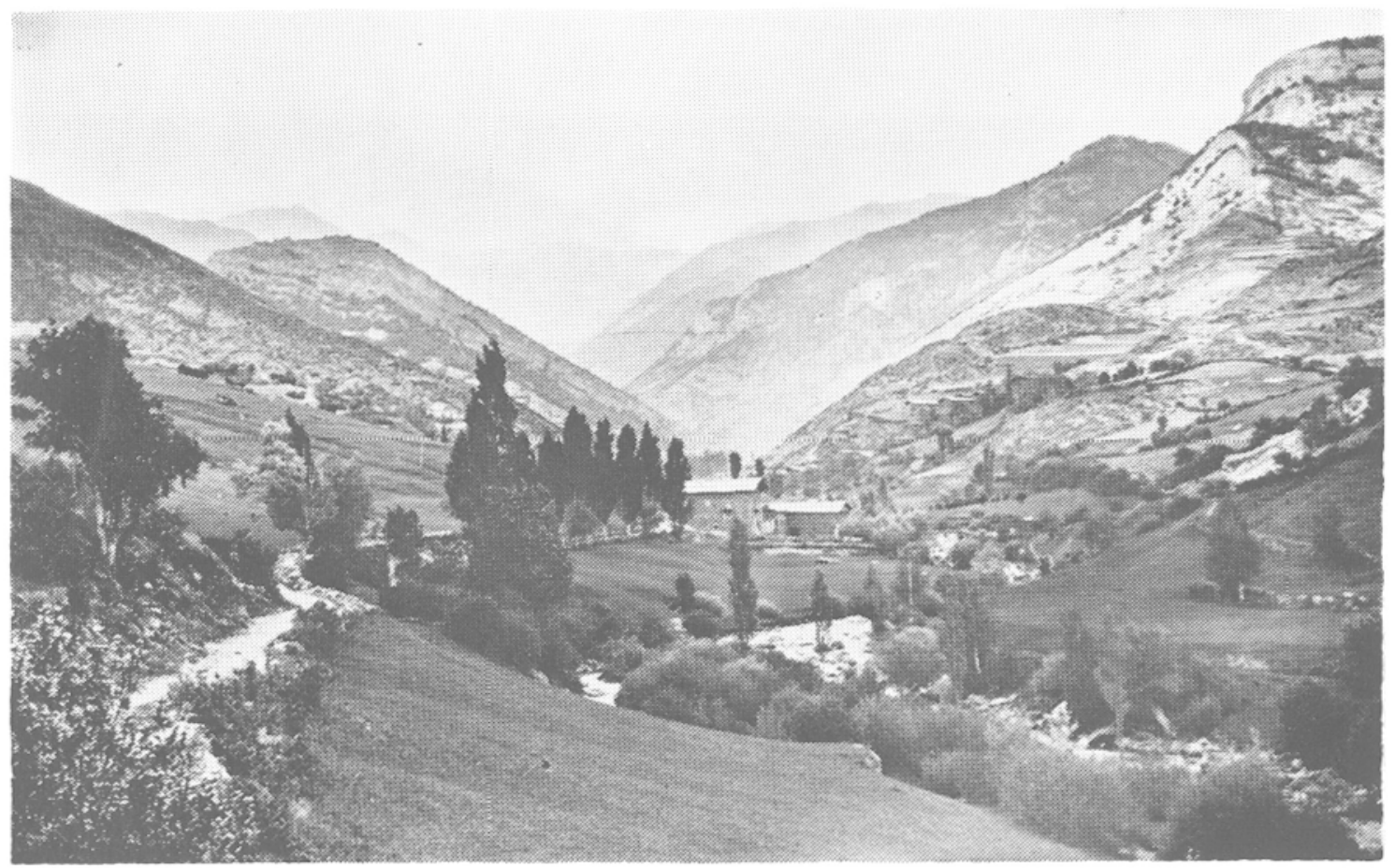

Photographie $\mathrm{n}^{\circ} 7$ : Vue de La Massana, août 1913 (cliché H. Gaussen).

portent sur le versant de l'obac de Soldeu, de l'autre côté de la crête, où se trouvent les installations de ski de Soldeu-El Tarter.

Plus en aval, le cliché ancien, qui montrait la petite chapelle de Les Bons et les ruines de la tour voisine, est surtout intéressant pour son arrière plan: l'obac d'Encamp, à l'entrée de la vallée dels Cortals. La comparaison entre les deux photographies (nos 5 et 6 ) est là encore fort instructive pour évoquer l'évolution de l'espace andorran au XX" siècle et les problèmes qu'elle pose.

Continuant de descendre la vallée, on est en effet arrivé là au niveau où les villages se trouvent étroitement enserrés entre les deux versants raides et les possibilités de mise en culture, autrefois, et d'urbanisation aujourd'hui, fort limitées ( $\mathrm{n}^{\mathrm{s}} 7$ et 8 ).

A l'arrière-plan du cliché de 1913, on distingue la division de l'exploitation du versant : dans le bas, d'assez larges parcelles bocagères, de teinte gris sombre, constituent les prairies de fauche irriguées, fondamentales pour l'élevage et dont les superficies ne firent qu'augmenter au XIX" siècle. Sur la gauche, les prairies continuent dans toute la vallée dels Cortals, au long des hameaux de bordes d'été. Le bocage de frênes émondés accompagne étroitement le terroir de prairie.

Sur le versant, dans la zone rocailleuse et sèche juste en dessous de la forêt, on note la position des cultures sèches en terrasses. Cette zone, qui correspond aux anciens éboulis post-glaciaires et aux ver- 


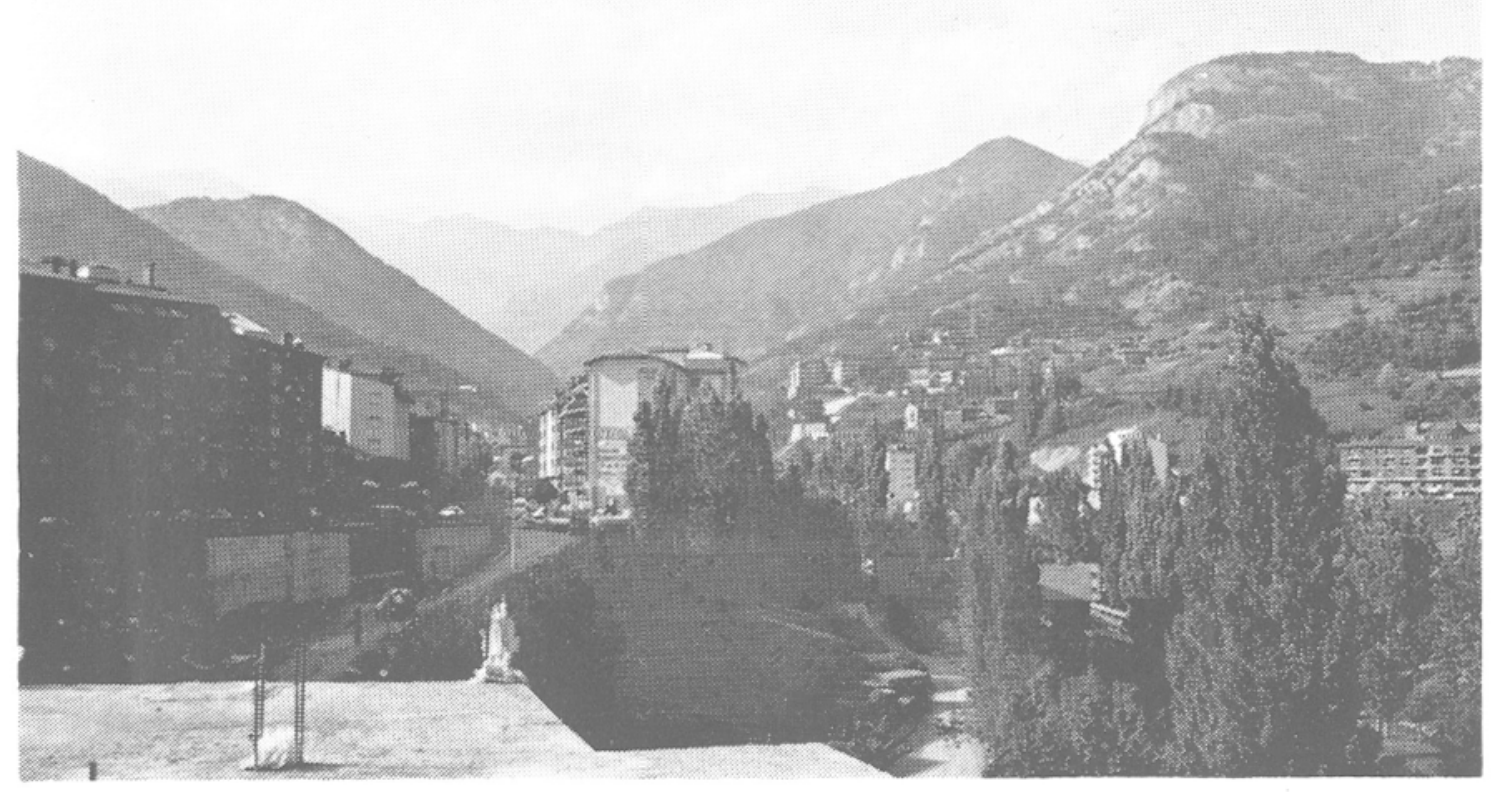

Photographie no 8: Vue de La Massana, septembre 1991 (cliché J.-P. Métailié).

sants de terrasses morainiques, est traversée par deux couloirs torrentiels actifs, nés dans la forêt de l'obac et à l'aspect très frais au moment de la photographie. Les écoulements torrentiels ont dû probablement correspondre aux crues enregistrées, d'autre part, en Ariège en 1910, 1911 et 1912 (1).

Avec la photographie de 1991 on discerne très bien les mutations de l'espace. L'urbanisation du versant est la plus évidente; les prairies subsistant au milieu des immeubles sont aujourd'hui cultivées en tabac; les anciennes pièces de cultures non urbanisées ont en revanche été abandonnées et sont colonisées par la pinède. Les couloirs torrentiels sont maintenant cachés par la végétation et les constructions, mais le principal est toujours actif et a provoqué de nombreux dégâts en novembre 1982, de même que le ruisseau dels Cortals, d'autant plus que l'urbanisation s'est étendue sur la zone d'épandage torrentiel. A l'arrière plan, la forêt de pins peu exploitée s'est densifiée sur tous les versants visibles.

Le village de la Massana, comme celui d'Ordino, a une situation un peu originale en Andorre: il n'est pas localisé dans un fond de vallée étroit mais dans une large cuvette, zone de confluence et d'épandage morainique des glaciers provenant du Valira del Nord et du Vall d'Arinsal.

(1) Sur le thème des écoulements dévastateurs dans les Pyrénées, se reporter à $R G P S O, 58,1,1987$, "Catastrophes ", et sur les crues arićgeoises du début de la décennié 1910 à J.-M. Antoine, Un torrent oublié... en Haute-Ariège, RGPSO, 59, 1, 1988, "Montagnes fragiles». 


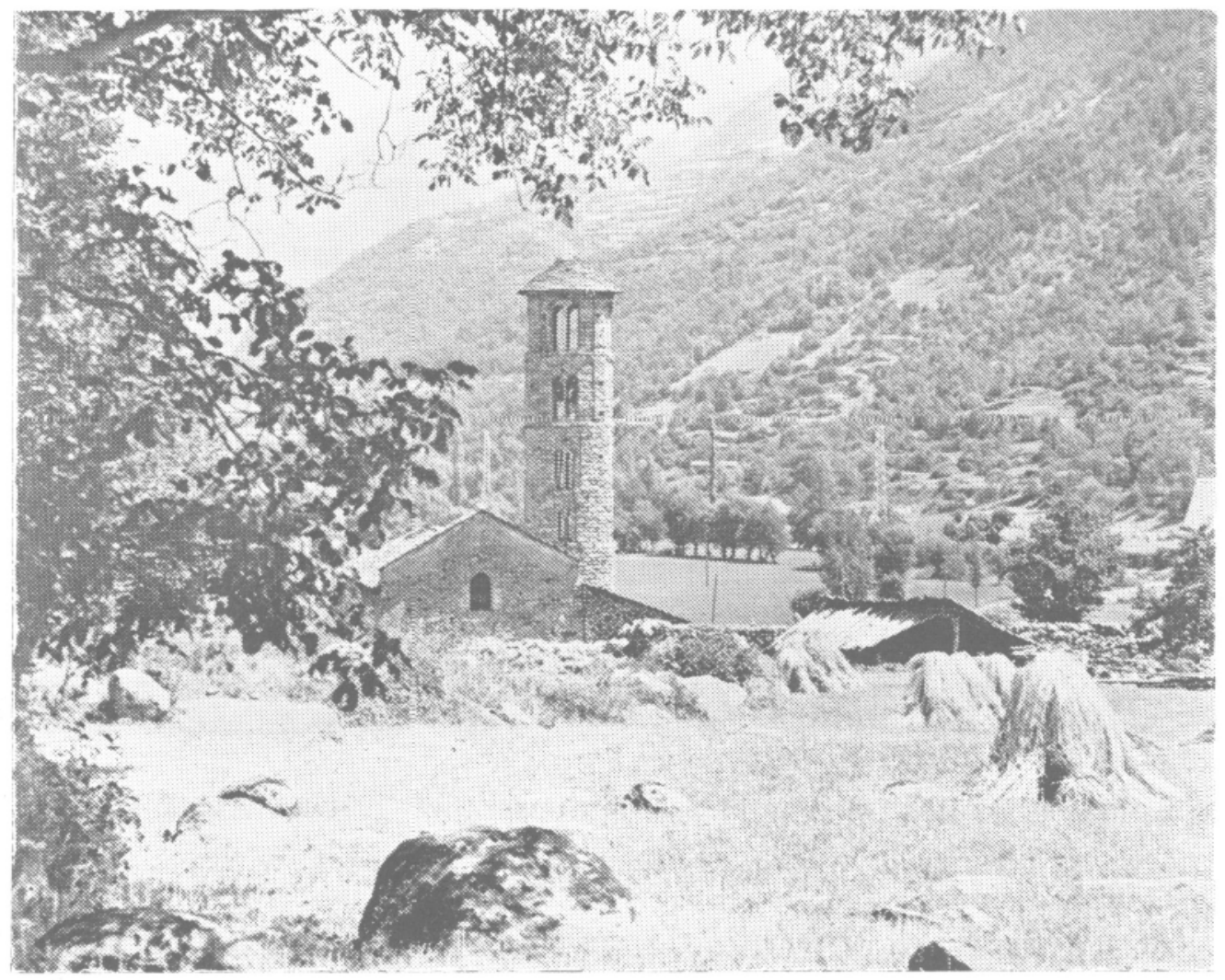

Photographie $\mathrm{n}^{\circ}$ 9: Eglise de Santa Coloma, juillet 1936 (cliché H. Gaussen).

Le cliché de 1913 révèle cet aspect particulier de formes topographiques douces, couvertes de prairies, quelques cultures s'étageant à droite sur les parties escarpées de la solana. Le paysage est ici fort différent de celui des basses vallées encaissées. Le village et ses hameaux eux-mêmes, qui sont à peine visibles dans les replis du terrain, ne sont pas bâtis sur le modèle des villages-rue coincés dans les fonds de vallée, caractéristique du Valira d'Orient, mais forment des noyaux.

L'urbanisation galopante est le fait essentiel que souligne la comparaison; La Massana, qui compte aujourd'hui 3678 habitants (2), est en tra:n de s'étendre de tous côtés sur des versants facilement accessibles et constructibles, sans risques torrentiels ou avalancheux; prairies et champs de tabac parsèment les vides entre les immeubles et les lotissements.

A l'arrière-plan, comme dans toutes les photos précédentes, on note la densification de la forêt et la colonisation des cultures abandon-

(2) Le récent recensentent de la population en Andorre fait d'ailleurs réssortir que près de $80 \%$ des habitants de La Massana sont étrangers : 38,3\% d'Espagnols, $12 \%$ de Portugais, $10 \%$ de Français, $11 \%$ d'Anglais et $8 \%$ de ressortissants d'autres nationalités. 


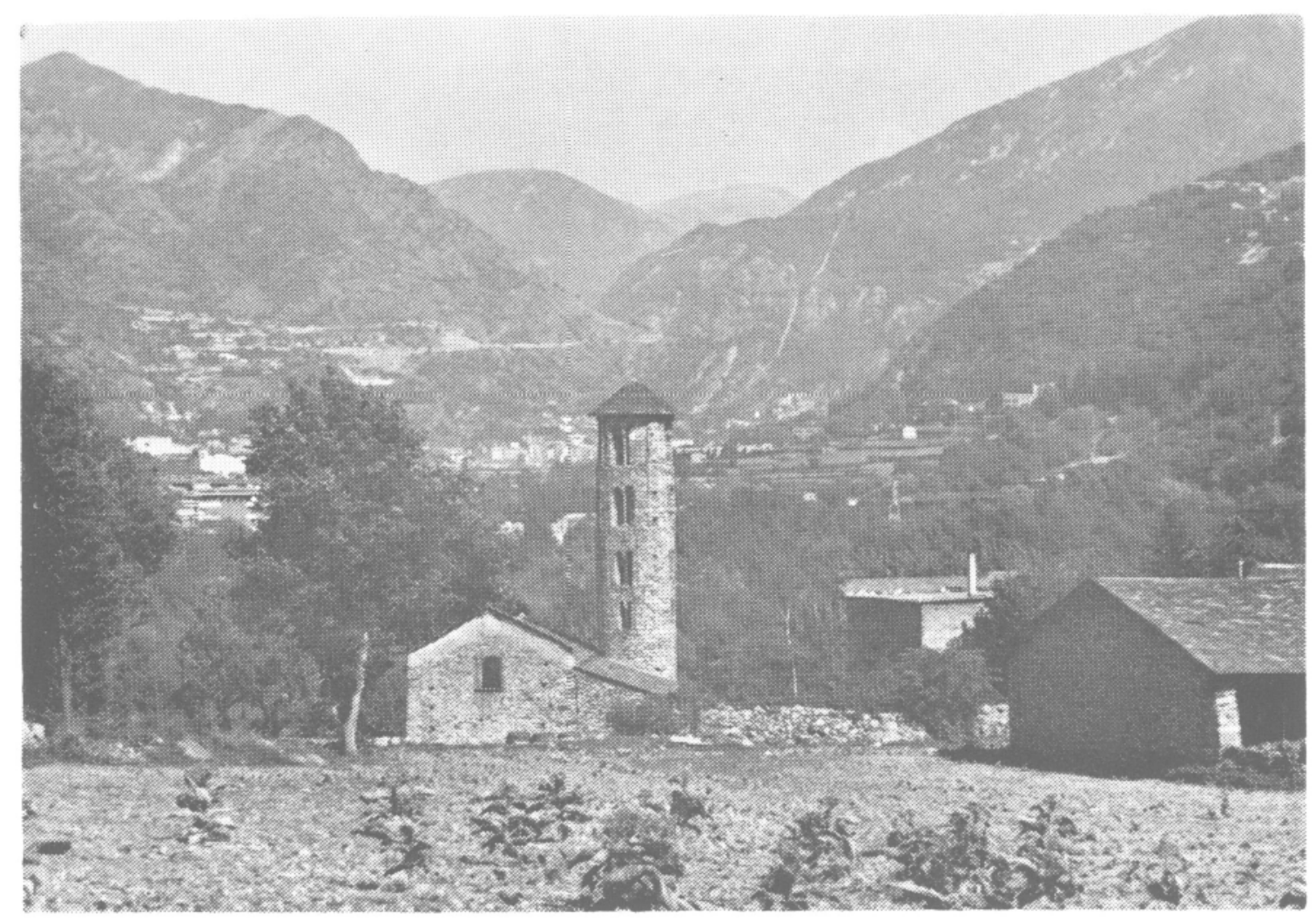

Photographie n" 10: Eglise de Santa Coloma, septembre 1991 (cliché J.-P. Métailié).

nées: en 1913, les versants n'étaient piquetćs quc d'une maigre brousse de chênes à la solana, de pins sylvestres à l'obac; la pinède est désormais en train de coloniser les deux expositions

L'église de Santa Coloma, un des plus vieux monuments d'Andorre, à l'aval d'Andorra, remonte aux $\mathrm{X}^{\mathrm{e}}$ et $\mathrm{XII}^{\mathrm{e}}$ siècles. L'axe de la prise de vue permet de conserver encore de nos jours une vision bucolique des alentours de l'église, où ne se devinent pas les immeubles, hôtels, lotissements, ni la route surchargée de circulation qui longe le site $\left(n^{\text {os }} 9\right.$ et 10$)$.

A l'arrière plan, la croissance des rideaux d'arbres produit un effet de masque qui oblitère l'urbanisation d'Andorra-la-Vel'la ; seuls quelques toits et grues de chantier en émergent.

Au premier plan, les moissons de 1936 ont cédé la place à l'omniprésent tabac.

MoTs-ClLÉs. - PyrÉNÉES, ANDORRE, paysages, collection de photographie Henri Gaussen, comparaisons de clichés en 1913 ou 1936 avec des clichés pris en 1991. 